\title{
Porous graphene and graphenylene nanotubes: Electronic structure and strain effects
}

\author{
Guilherme S.L. Fabris $^{\mathrm{a}, 1}$, Chad E. Junkermeier ${ }^{\mathrm{b}}$, Ricardo Paupitz ${ }^{\mathrm{a}, *}$ \\ a São Paulo State University (UNESP), Institute of Geosciences and Exact Sciences, Rio Claro, 13506-900 SP, Brazil \\ ${ }^{\mathrm{b}}$ Research Corporation of the University of Hawaii'i, Honolulu, HI 96848, United States
}

\section{A R T I C L E I N F O}

\section{Article history:}

Received 9 June 2017

Received in revised form 16 August 2017

Accepted 4 September 2017

Available online 15 September 2017

\section{Keywords:}

Carbon nanotubes

Porous graphene

Graphenylene

Simulation

Strain effects

Porous nanotubes

\begin{abstract}
A B S T R A C T
The unusual and unique mechanical and electronic properties of nanostructured carbon materials make them useful in the construction of nanodevices. We investigate a new class of structures, called porous nanotubes, which are constructed from two recently synthesized two-dimensional materials, namely the porous graphene (PG) and the two-dimensional carbon allotrope known as graphenylene, also known as Biphenylene Carbon (BPC). We investigate this class of quasi-one-dimensional materials using the density functional tight-binding (DFTB) method to optimize geometries and to calculate electronic structure features of these systems. For each type of porous nanotube, calculations were performed on tubes with several diameters and chiralities. Our results show that the PG nanotubes have a wide band-gap, $\sim 3.3 \mathrm{eV}$, and the graphenylene nanotubes have a semiconductor behavior with a band gap around $0.7 \mathrm{eV}$. They also show that as the diameter of a PG nanotube increases the band-gap decreases, while for the graphenylene nanotube the band gap increases. In both cases, the observed gap variation with increasing diameter is towards the value found for the respective two-dimensional membrane. Calculations on axially strained porous nanotubes show a decrease on the band gap of $\sim 10 \%$ for some chiralities of the PG nanotube and an increase for the graphenylene nanotubes gap that can become as high as $100 \%$. These results are in contrast with the expected behavior for carbon nanotubes, which show a linear dependence between gap opening and applied strain under similar conditions.
\end{abstract}

(c) 2017 Elsevier B.V. All rights reserved.

\section{Introduction}

Graphene [1,2] is a two-dimensional array of hexagonal units of $\mathrm{sp}^{2}$ bonded carbon atoms (Fig. 1) which presents unusual and interesting electronic and mechanical properties [2]. Because of these properties it may be used in diverse applications, for example electronics [3,4], water separation [5], nanomechanical resonators [6-8], chemical sensors [9], or in producing exotic materials [1014]. However, in its pristine form, graphene is a gapless semiconductor. This characteristic imposes serious limitations to its use in practical electronic applications. Many approaches have been proposed to create a gap in graphene-like materials. One of the most common strategies uses chemisorption methods, such as oxidation [15-17], hydrogenation [18-20], fluorination [21-24], or some other adsorbate $[9,25]$. Another approach is to obtain intrinsically hydrogenated structures, such as PG (Fig. 1b), whose syn-

\footnotetext{
* Corresponding author.

E-mail address: paupitz@rc.unesp.br (R. Paupitz).

1 Present address: Grupo de Modelagem e Simulação Molecular - DM, São Paulo State University - UNESP, Caixa Postal 473, Bauru - São Paulo, SP, Brazil.
}

thesis was recently achieved [26]. Other authors discussed the metallic behavior found for structures obtained by the combination of biphenylenes in several configurations [27] which, despite some structural similarities, are different from the structures discussed in the present work, which can present semiconducting or insulating behavior. One of these combinations is the so called graphenylene, which has a small band gap and delocalized frontier orbitals [28-30]. A possible route to the synthesis of graphenylene was proposed elsewhere [28] and its experimental realization, although obtained through a different route, was reported recently [31,32]. Further, Schlütter et al. reported recently that they were able to synthesize octafunctionalized biphenylene GNRs [33]. Another possibility is the combination of these geometries with functionalization [34] that can lead to interesting effects regarding gas adsorption [35]. The new generation of electronics may also be facilitated by producing any of the above structures using boron nitride (BN) or some combination of carbon, boron, and nitrogen atoms [36,37]. In this context, we consider the possibility of structures based on the PG and graphenylene primitive cells and on CNT architecture, the so-called porous nanotubes (PNT). The graphenylene version of the PNTs was proposed recently by Koch et al. [38] 


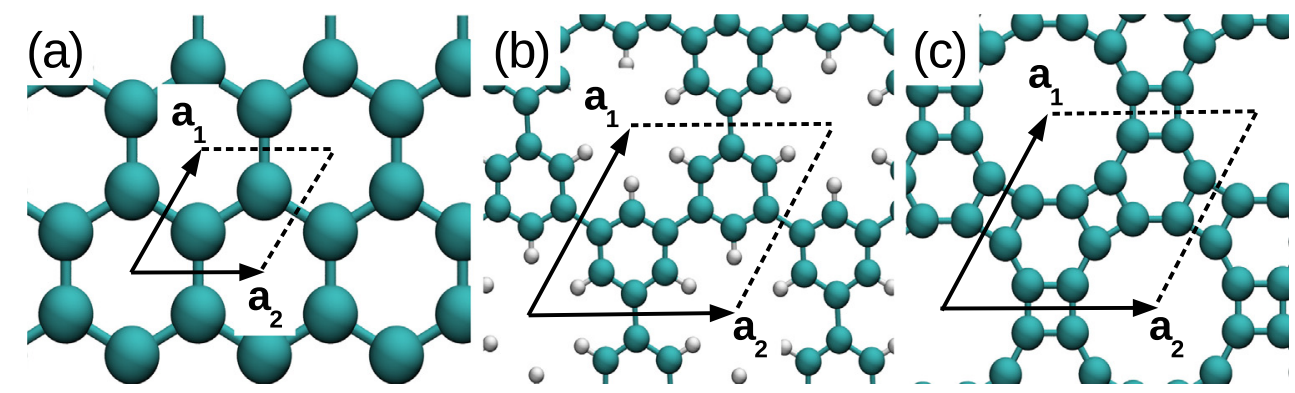

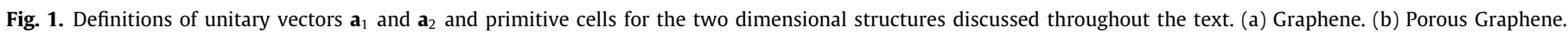
(c) Bi-Phenylene Carbon.

and, taking into account the fact that density functional theory (DFT) calculations tends to underestimate gap openings, our results are in good agreement with their findings. In the present work, we also study gap variations caused by axial strain on the proposed structures. In order to investigate structural and electronic properties of this kind of material we investigate sixty-two representative one-dimensional structures. These are based on nine armchair-like, fourteen zigzag-like, and eight chiral-like PGbased PNTs with or corresponding graphenylene-based PNTs. Using DFTB we computed the electronic structure and studied the structural properties of each one of these structures.

\section{Methods}

Geometry optimizations and electronic structure calculations of planar structures and PNTs were performed using a selfconsistent-charge (SCC) version of the density functional tightbinding (DFTB) method [39-41] in the DFTB+ code [42]. This methodology can be implemented in a low computational cost code and mixes advantages of tight binding methods and of DFT precision in the description of molecular and condensed matter systems [43]. This methodology is based on a second-order expansion of the Kohn-Sham energy defined in DFT [40,44]. Band gap values predicted by DFTB may be underestimated, caused by the formulation being based only on valence electrons, yet it is useful in obtaining relative energy values or to explain trends in a series of large atomic structures. The particular parameterization used in the present work, pbc $[45,46]$ Slater-Koster files, was developed for solids and surfaces and has been used in modeling systems similar to those discussed here [13]. Convergence criteria for the geometry optimization used force differences of $10^{-5}$ with a SCC tolerance of $10^{-4}$. For sampling the points in the Brillouin zone, we used a $10 \times 1 \times 1$ Monkhorst-Pack grid [47] in which the 10 folding is in the direction related to the axis of the PNT. Also, as vacuum condition, it was considered a 50 Ådistance of empty space in directions perpendicular to the nanotube axis. The molecular dynamics (MD) simulations discussed in the present work were carried out using the Verlet algorithm, while temperatures were controlled through a Berendsen thermostat.

\section{Results and discussion}

\subsection{From membranes to tubes}

In order to define a systematic way for the construction of the PNTs, we took advantage of the geometrical similarities of the unit cells when comparing PG and graphenylene membranes with graphene. Using the well known definition of chiral indices for CNTs $[48,49]$ we define a chiral vector for each one of our template planar PG and graphenylene membranes. The construction of PNTs
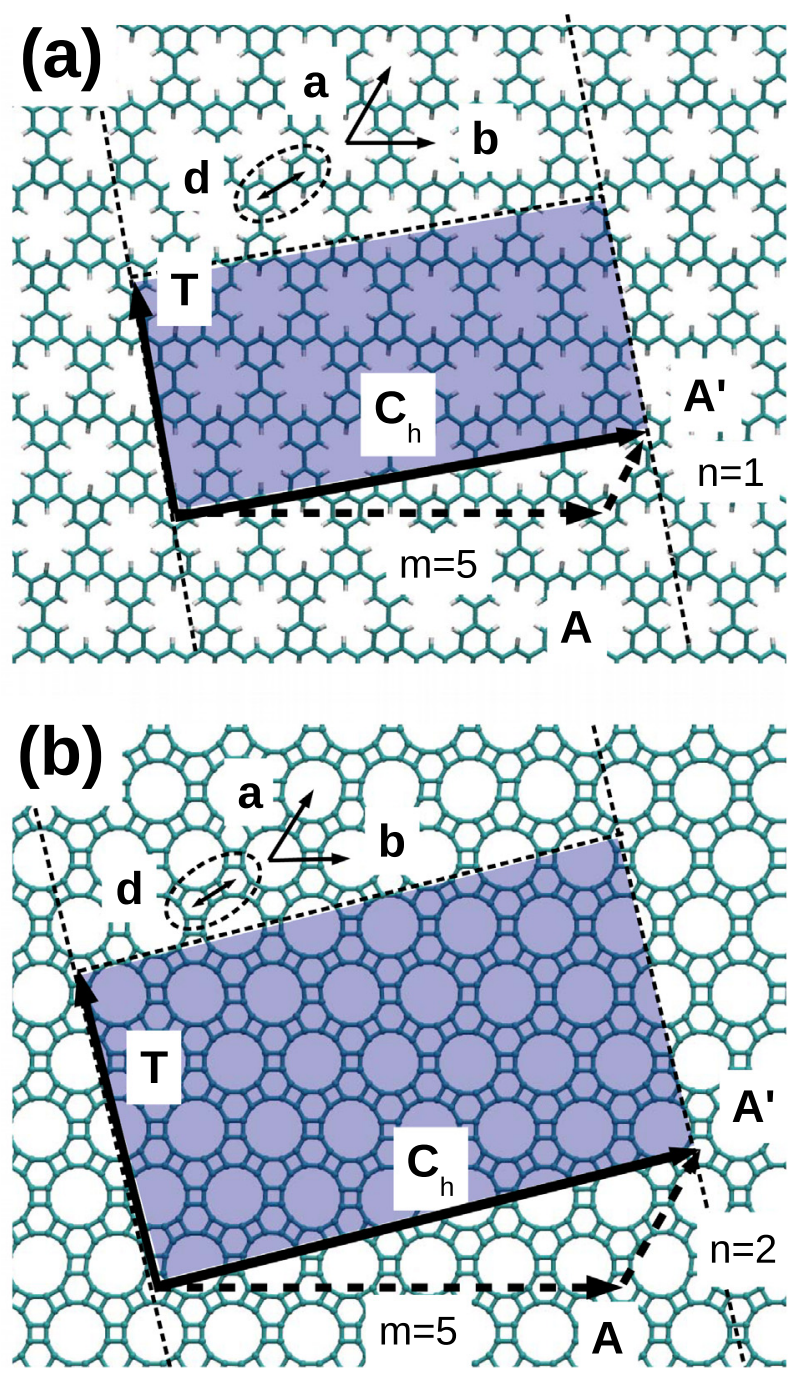

Fig. 2. Geometrical definitions and chiral indices for two-dimensional structures considered in this work. The vectors $\mathbf{a}$ and $\mathbf{b}$ are the unit vectors of the PG and graphenylene sheets. The vectors $\mathbf{A}$ and $\mathbf{A}^{\prime}$ are respectively multiples of $\mathbf{a}$ and $\mathbf{b}$ used in defining the chiral vector $\mathbf{C}_{\mathbf{h}}$, with $\mathbf{T}$ being the translation vector. Shaded areas indicate the unrolled primitive cell for specific examples of chiralities. Eliptic regions delimited by dashed lines indicate the distance $\mathbf{d}$. (a) PG with primitive cell of a $(5,1)$ PNT indicated by shaded area. (b) Graphenylene sheet with primitive cell of a $(5,2)$ PNT indicated by the shaded area.

follow a recipe similar to that used in the case of the usual carbon nanotubes. In Fig. 2, chiral indices $n$ and $m$ are defined for both, PG (Fig. 2a) and graphenylene (Fig. 2b) PNTs as well as their chiral vector $\mathbf{C}_{h}$, primitive lattice vectors, translational vector $\mathbf{T}$ and quasi 
Table 1

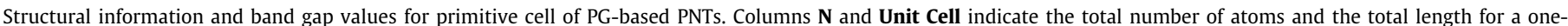

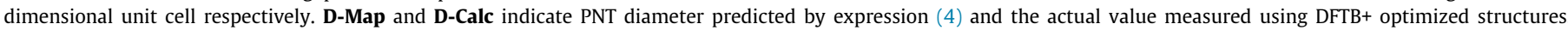

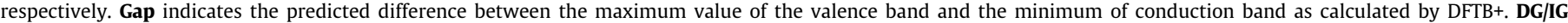
indicates if the gap is direct or indirect.

\begin{tabular}{|c|c|c|c|c|c|c|}
\hline$(m, n)$ & $N$ & Unit Cell $(\AA ̊)$ & D-Map $(\AA ̊)$ & D-Calc $(\AA)$ & Gap (eV) & $\mathrm{DG} / \mathrm{IG}$ \\
\hline$(2,2)$ & 72 & 7.439 & 8.42 & 7.89 & 3.326 & IG \\
\hline$(3,3)$ & 108 & 7.443 & 12.63 & 12.21 & 3.368 & IG \\
\hline$(4,4)$ & 144 & 7.449 & 16.84 & 16.19 & 3.367 & IG \\
\hline$(5,5)$ & 180 & 7.453 & 21.06 & 21.24 & 3.357 & IG \\
\hline$(6,6)$ & 216 & 7.456 & 25.27 & 24.47 & 3.352 & IG \\
\hline$(7,7)$ & 252 & 7.459 & 29.48 & 29.23 & 3.34 & IG \\
\hline$(8,8)$ & 288 & 7.462 & 33.69 & 32.73 & 3.333 & IG \\
\hline$(9,9)$ & 324 & 7.463 & 37.9 & 37.18 & 3.337 & IG \\
\hline$(10,10)$ & 360 & 7.464 & 42.11 & 41.29 & 3.324 & IG \\
\hline$(2,0)$ & 72 & 12.473 & 4.86 & 4.87 & 3.033 & DG \\
\hline$(3,0)$ & 108 & 12.647 & 7.29 & 7.19 & 3.435 & DG \\
\hline$(4,0)$ & 144 & 12.731 & 9.73 & 10.08 & 3.434 & DG \\
\hline$(5,0)$ & 180 & 12.773 & 12.16 & 12.03 & 3.421 & DG \\
\hline$(6,0)$ & 216 & 12.802 & 14.59 & 14.79 & 3.406 & DG \\
\hline$(7,0)$ & 252 & 12.819 & 17.02 & 17.67 & 3.395 & DG \\
\hline$(8,0)$ & 288 & 12.835 & 19.45 & 19.53 & 3.383 & DG \\
\hline$(9,0)$ & 324 & 12.847 & 21.88 & 22.32 & 3.372 & DG \\
\hline$(10,0)$ & 360 & 12.857 & 24.31 & 24.27 & 3.365 & DG \\
\hline$(11,0)$ & 396 & 12.865 & 26.74 & 26.05 & 3.357 & DG \\
\hline$(12,0)$ & 432 & 12.872 & 29.18 & 29.01 & 3.351 & DG \\
\hline$(13,0)$ & 468 & 12.878 & 31.61 & 31.72 & 3.346 & DG \\
\hline$(14,0)$ & 504 & 12.884 & 34.04 & 33.76 & 3.341 & DG \\
\hline$(15,0)$ & 540 & 12.888 & 36.47 & 36.25 & 3.34 & DG \\
\hline$(2,1)$ & 252 & 33.911 & 6.43 & 6.33 & 3.338 & IG \\
\hline$(3,1)$ & 468 & 46.129 & 8.77 & 9.31 & 3.404 & IG \\
\hline$(4,1)$ & 252 & 19.555 & 11.14 & 11.09 & 3.415 & IG \\
\hline$(4,2)$ & 504 & 34.04 & 12.87 & 12.63 & 3.388 & IG \\
\hline$(5,2)$ & 468 & 26.776 & 15.18 & 14.55 & 3.389 & IG \\
\hline$(6,2)$ & 936 & 46.38 & 17.53 & 17.38 & 3.38 & IG \\
\hline$(8,2)$ & 504 & 19.66 & 22.28 & 21.65 & 3.37 & IG \\
\hline$(12,2)$ & 3096 & 84.5 & 31.89 & 31.49 & 3.34 & IG \\
\hline
\end{tabular}

Table 2

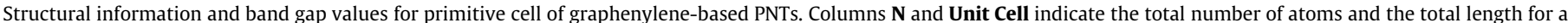

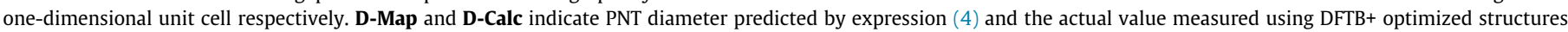

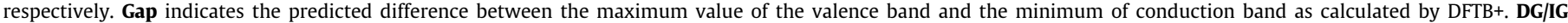
indicates if the gap is direct or indirect.

\begin{tabular}{|c|c|c|c|c|c|c|}
\hline$(m, n)$ & $N$ & Unit Cell $(\AA)$ & D-Map $(\AA ̊)$ & D-Calc $(\AA)$ & Gap (eV) & $\mathrm{DG} / \mathrm{IG}$ \\
\hline$(2,2)$ & 48 & 6.777 & 7.39 & 7.56 & 0.494 & DG \\
\hline$(3,3)$ & 72 & 6.781 & 11.08 & 11.08 & 0.626 & DG \\
\hline$(4,4)$ & 96 & 6.782 & 14.77 & 14.86 & 0.675 & DG \\
\hline$(5,5)$ & 120 & 6.783 & 18.46 & 18.62 & 0.697 & DG \\
\hline$(6,6)$ & 144 & 6.783 & 22.16 & 22.38 & 0.709 & DG \\
\hline$(7,7)$ & 168 & 6.784 & 25.85 & 26.12 & 0.717 & DG \\
\hline$(8,8)$ & 192 & 6.784 & 29.54 & 29.87 & 0.721 & DG \\
\hline$(9,9)$ & 216 & 6.784 & 33.23 & 33.11 & 0.725 & DG \\
\hline$(10,10)$ & 240 & 6.784 & 36.93 & 37.34 & 0.727 & DG \\
\hline$(2,0)$ & 48 & 11.986 & 4.26 & 4.24 & 0.688 & DG \\
\hline$(3,0)$ & 72 & 11.856 & 6.4 & 6.46 & 0.443 & DG \\
\hline$(4,0)$ & 96 & 11.811 & 8.52 & 8.24 & 0.693 & DG \\
\hline$(5,0)$ & 120 & 11.789 & 10.66 & 10.79 & 0.709 & DG \\
\hline$(6,0)$ & 144 & 11.778 & 12.79 & 12.69 & 0.655 & DG \\
\hline$(7,0)$ & 168 & 11.771 & 14.92 & 15.11 & 0.719 & DG \\
\hline$(8,0)$ & 192 & 11.766 & 17.06 & 17.07 & 0.724 & DG \\
\hline$(9,0)$ & 216 & 11.763 & 19.19 & 19.43 & 0.699 & DG \\
\hline$(10,0)$ & 240 & 11.761 & 21.32 & 21.43 & 0.728 & DG \\
\hline$(11,0)$ & 264 & 11.759 & 23.45 & 23.75 & 0.729 & DG \\
\hline$(12,0)$ & 288 & 11.758 & 25.58 & 25.78 & 0.715 & DG \\
\hline$(13,0)$ & 312 & 11.757 & 27.71 & 28.07 & 0.731 & DG \\
\hline$(14,0)$ & 336 & 11.756 & 29.85 & 30.23 & 0.732 & DG \\
\hline$(15,0)$ & 360 & 11.756 & 31.98 & 32.26 & 0.723 & DG \\
\hline$(2,1)$ & 168 & 31.132 & 5.64 & 5.49 & 0.612 & IG \\
\hline$(3,1)$ & 312 & 42.485 & 7.69 & 7.6 & 0.691 & IG \\
\hline$(4,1)$ & 168 & 17.995 & 9.77 & 9.77 & 0.598 & IG \\
\hline$(4,2)$ & 336 & 31.105 & 11.28 & 11.22 & 0.708 & IG \\
\hline$(5,2)$ & 312 & 24.479 & 13.31 & 13.49 & 0.659 & IG \\
\hline$(6,2)$ & 624 & 42.39 & 15.37 & 15.67 & 0.72 & DG \\
\hline$(8,2)$ & 336 & 17.961 & 19.54 & 20.01 & 0.701 & DG \\
\hline$(12,2)$ & 2064 & 77.09 & 27.96 & 28.56 & 0.731 & DG \\
\hline
\end{tabular}


one-dimensional primitive cells used in the construction of singlewall PNTs, each of which will be discussed further hereafter. The vector $\mathbf{C}_{h}$ indicates two equivalent sites on the sheet (PG or graphenylene) and is uniquely defined by a pair of integer numbers $(m, n)$,

$\mathbf{C}_{h}=m \mathbf{a}+n \mathbf{b}$,

where vectors a and b (Fig. 2) are the lattice's unit vectors for each case. Vector T, which indicates the axial direction of the PNT, is perpendicular to $\mathbf{C}_{h}$ and its length is defined by the distance between the original site (the origin of $\mathbf{C}_{h}$, for example) and its closest equivalent site. Rolling up the equivalent sites one over the other, forms the tube uniquely identified by the pair of integer numbers $(m, n)$. As with CNTs, when $m=n$ the PNT is denoted as armchair-type, those with $m=0$ or $n=0$ are the so-called zig-zag tubes, while other combinations correspond to chiral tubes.

In the case of CNTs, if one assumes that the known geometrical relations for two dimensional graphene membranes remain valid after wrapping, the exact number of atoms of the primitive cell of a nanotube is completely determined by its chiral indices $(m, n)[48,49]$. In a similar way, geometrical relations are straightforwardly obtained for PNTs, such as the extension of a primitive cell, indicated by shaded areas in Fig. 2. It is also possible to find mathematical expressions for the number of atoms in each one of these primitive cells. In the case of PG based PNTs, the number of carbon atoms $\left(n_{C}\right)$, the number of hydrogen atoms $\left(n_{H}\right)$ and total number of atoms $\left(n_{T}\right)$ are

$n_{C}=24 \cdot \frac{\left(n^{2}+m^{2}+n m\right)}{d_{R}}, n_{H}=12 \cdot \frac{\left(n^{2}+m^{2}+n m\right)}{d_{R}}, n_{T}=n_{C}+n_{H}$,

where $d_{R}$ is the maximum common divisor of $(2 n+m, 2 m+n)$. Analogously, for graphenylene-based PNTs one can find

$n_{C}=24 \cdot \frac{\left(n^{2}+m^{2}+n m\right)}{d_{R}}$.

The diameter, D, of an ideal CNT [48,49] as a function of $n$ and $m$ also has an analogous form for PNTs,

$D=\frac{\sqrt{n^{2}+m^{2}+n m}}{\pi} a$,

where $a$ indicates the length of the unit vectors of the twodimensional lattice (PG or graphenylene) and its magnitude depends on the distance $d$ between hexagons in the specific structure by the relation $a=\sqrt{3} d$.

\subsection{Structural properties}

Before employing the rolling-up procedure discussed above we first optimized PG and graphenylene. The optimization of the membranes resulted in a unit cell with lattice parameters a and $\mathrm{b}$ for PG and graphenylene with values 7.61, 7.48, 6.78 and $6.78 \AA$, respectively. This also resulted in distances between hexagons of $3.87 \AA$ and $4.41 \AA$ for graphenylene and PG respectively. With the optimized graphenylene and PG lattice vectors computed we created PG-based and graphenylene-based PNTs. Tables 1 and 2 present the lattice parameters, number of atoms, predicted and observed diameters, and band gaps of the PG-based and graphenylene-based PNTs. Using several pairs of diametrically opposed atoms through out an optimized tube we calculated the average value for the diameter of each PNT. As expected, there are some systematic differences between the diameter values

Table 3

Comparison between CNT total energies per atom with the energies found for PG $\left(\mathrm{E}_{\mathrm{PG}}\right)$ and graphenylene $\left(E_{B P C}\right)$ PNTs for various different chiralities.

\begin{tabular}{|c|c|c|c|}
\hline$(m, n)$ & $E_{C N T}(\mathrm{eV} /$ atom $)$ & $E_{P G}(\mathrm{eV} /$ atom $)$ & $E_{B P C}(\mathrm{eV} /$ atom $)$ \\
\hline$(2,2)$ & -46.288 & -34.653 & -46.488 \\
\hline$(3,3)$ & -46.869 & -34.672 & -46.542 \\
\hline$(4,4)$ & -47.087 & -34.677 & -46.561 \\
\hline$(5,5)$ & -47.185 & -34.680 & -46.569 \\
\hline$(6,6)$ & -47.238 & -34.680 & -46.574 \\
\hline$(7,7)$ & -47.269 & -34.681 & -46.577 \\
\hline$(8,8)$ & -47.290 & -34.681 & -46.579 \\
\hline$(9,9)$ & -47.304 & -34.681 & -46.580 \\
\hline$(10,10)$ & -47.314 & -34.681 & -46.581 \\
\hline$(2,0)$ & -45.430 & -34.564 & -46.277 \\
\hline$(3,0)$ & -46.112 & -34.638 & -46.454 \\
\hline$(4,0)$ & -46.588 & -34.661 & -46.512 \\
\hline$(5,0)$ & -46.846 & -34.670 & -46.538 \\
\hline$(6,0)$ & -46.997 & -34.675 & -46.552 \\
\hline$(7,0)$ & -47.097 & -34.677 & -46.561 \\
\hline$(8,0)$ & -47.158 & -34.679 & -46.567 \\
\hline$(9,0)$ & -47.198 & -34.680 & -46.570 \\
\hline$(10,0)$ & -47.230 & -34.680 & -46.573 \\
\hline$(11,0)$ & -47.252 & -34.681 & -46.575 \\
\hline$(12,0)$ & -47.268 & -34.681 & -46.577 \\
\hline$(13,0)$ & -47.282 & -34.681 & -46.578 \\
\hline$(14,0)$ & -47.292 & -34.681 & -46.579 \\
\hline$(15,0)$ & -47.299 & -34.681 & -46.580 \\
\hline$(2,1)$ & -46.155 & -34.626 & -46.419 \\
\hline$(3,1)$ & -46.382 & -34.656 & -46.495 \\
\hline$(4,1)$ & -46.747 & -34.668 & -46.529 \\
\hline$(4,2)$ & -46.900 & -34.672 & -46.543 \\
\hline$(5,2)$ & -47.024 & -34.676 & -46.555 \\
\hline$(6,2)$ & -47.114 & -34.678 & -46.562 \\
\hline$(8,2)$ & -47.204 & -34.680 & -46.571 \\
\hline$(12,2)$ & -47.283 & -34.681 & -46.578 \\
\hline
\end{tabular}

(b)
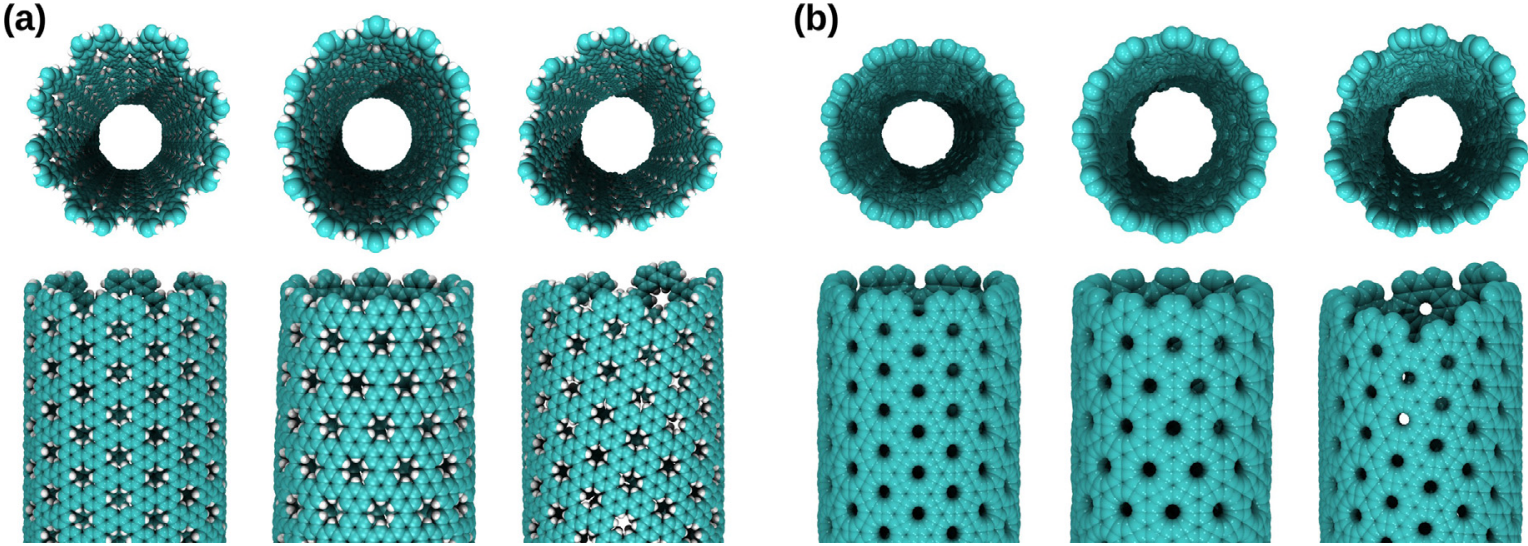

Fig. 3. Geometry of representative PNTs with chirality $(8,8),(8,0)$ and $(8,2)$. (a) Porous Graphene based. (b) Graphenylene-based. 

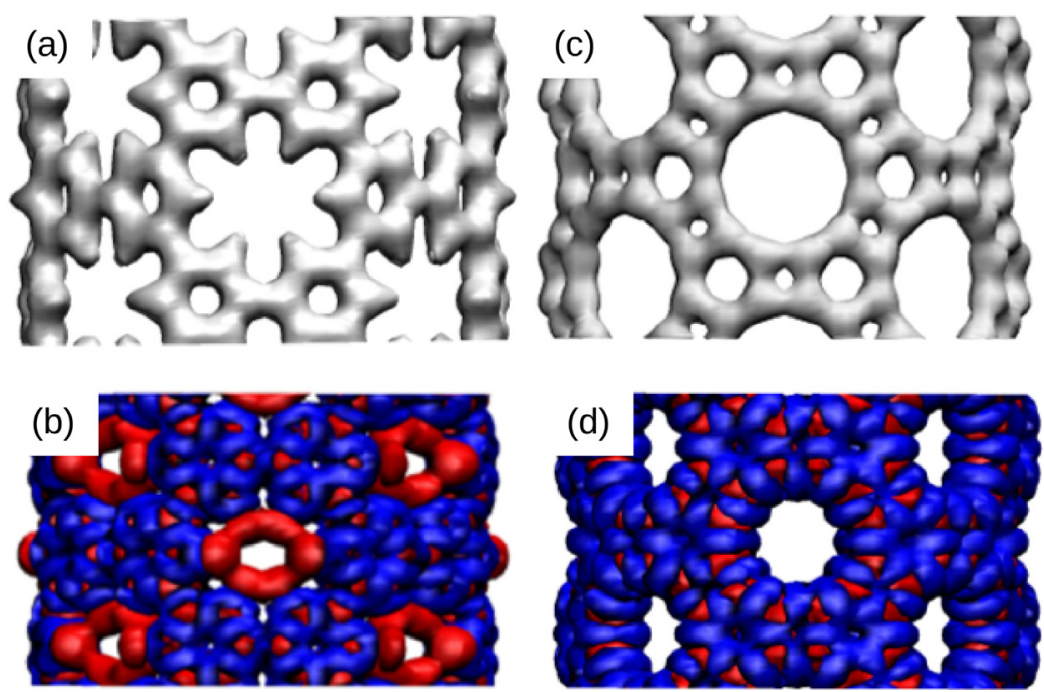

Fig. 4. Charge distributions for PG and graphenylene-based PNTs, both with chiral indices $(4,4)$. (a) and (b) Illustrate charge and charge differences for a PG based PNT, respectively. (c) and (d) Are respectively the charge distribution and charge differences found for a graphenylene-based PNT.

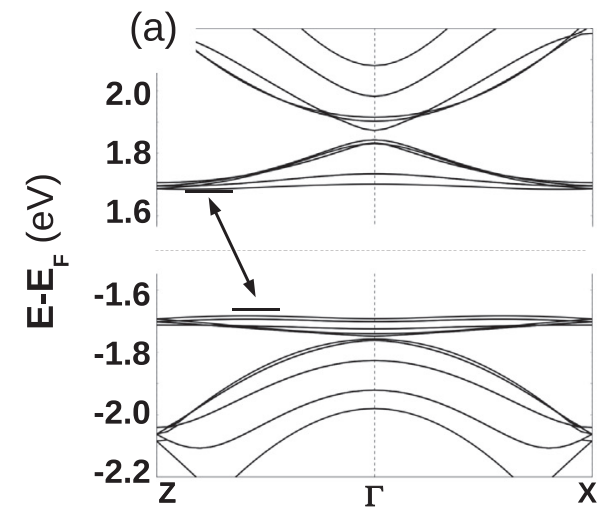

(c)
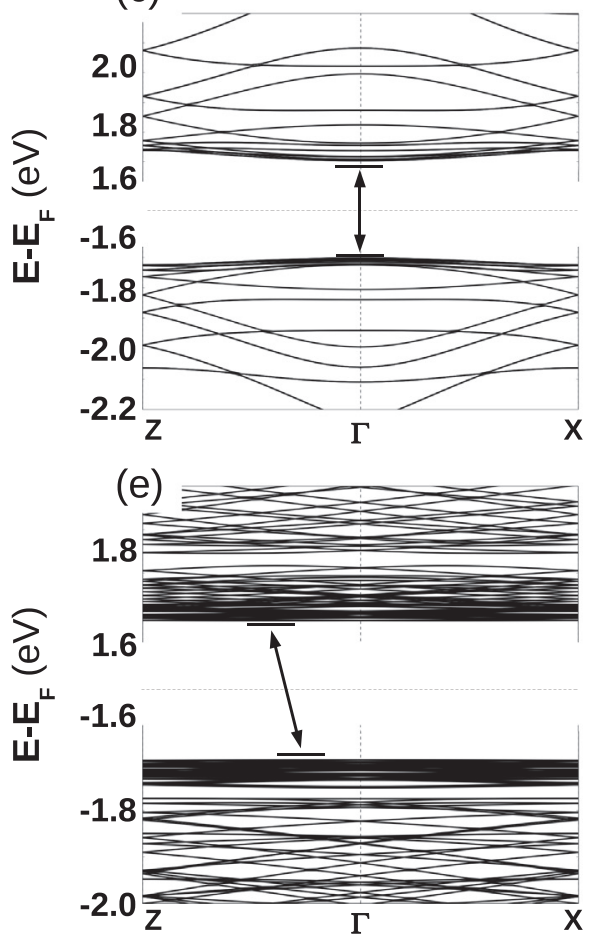

(b)

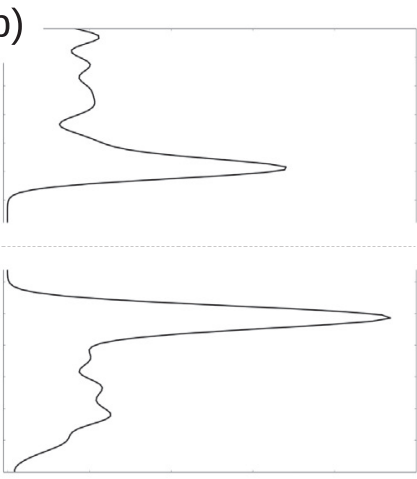

(d)
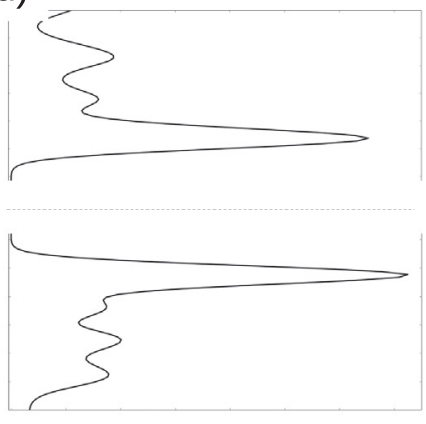

$(\mathrm{f})$
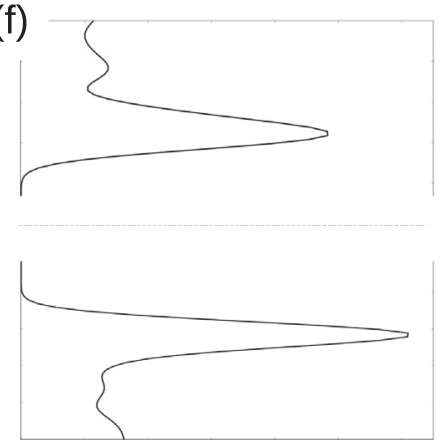

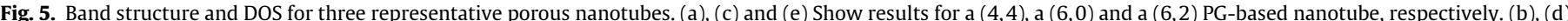

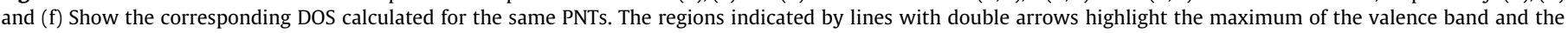
minimum of the conduction band for each case. Whether these are vertically aligned or not defines if the gap is named as being direct or indirect respectively. 

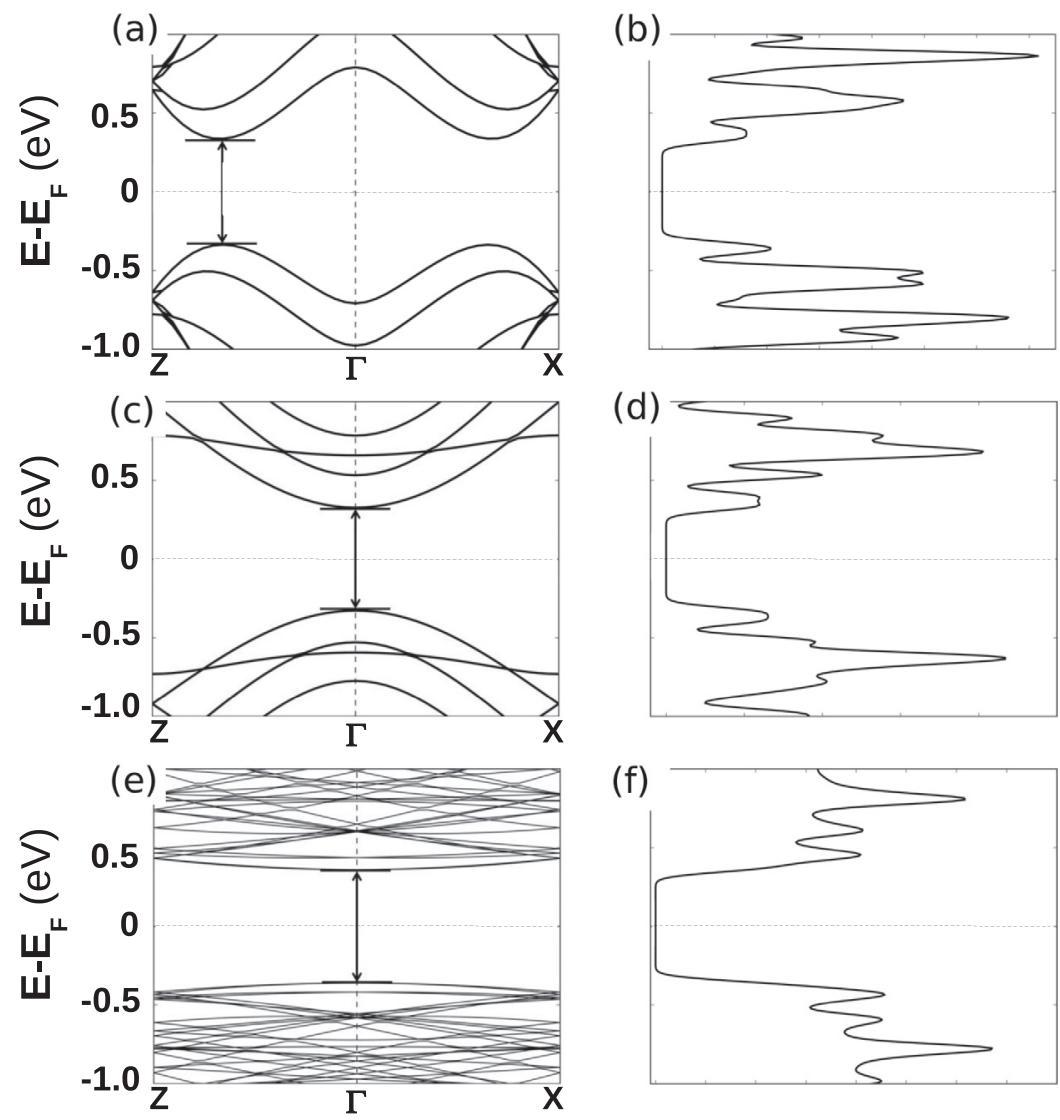

(f)

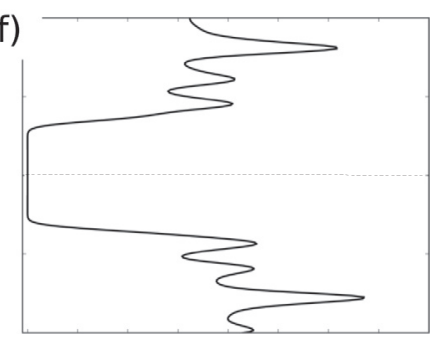

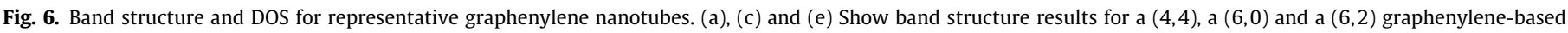
nanotube respectively. (b), (d) and (f) Show the DOS calculated for the same PNTs, namely, a $(4,4)$, a $(6,0)$ and a $(6,2)$ respectively.

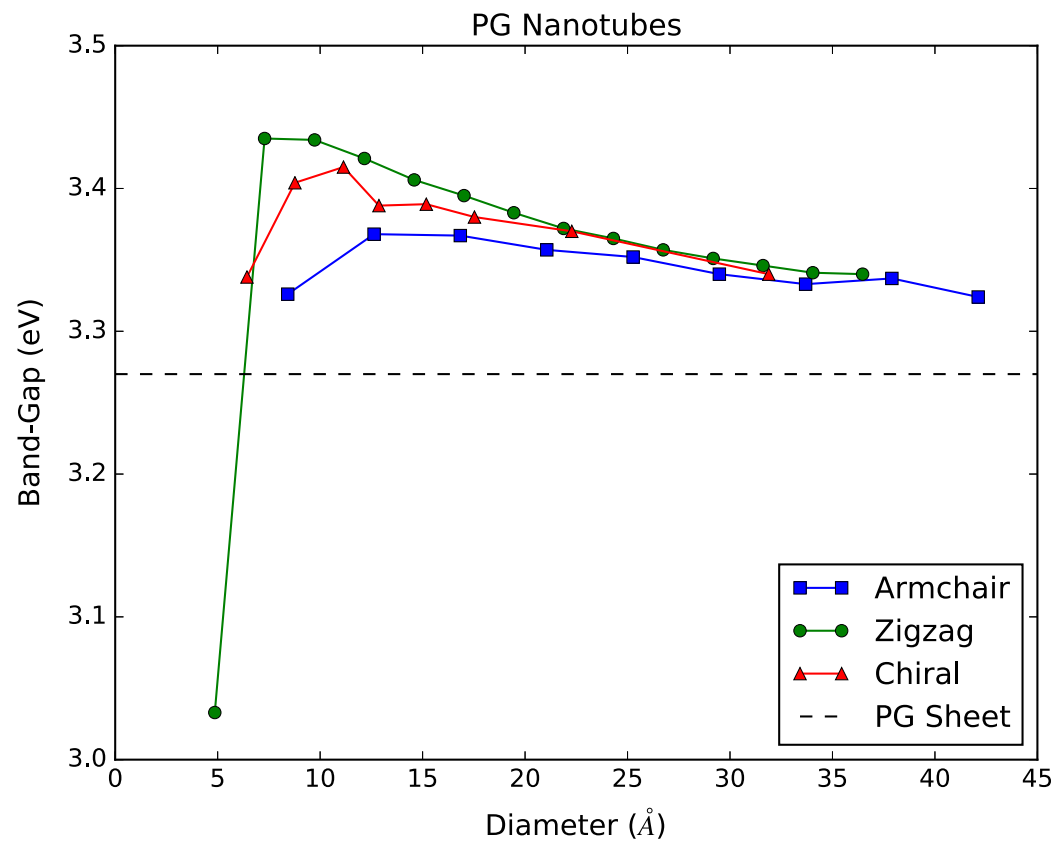

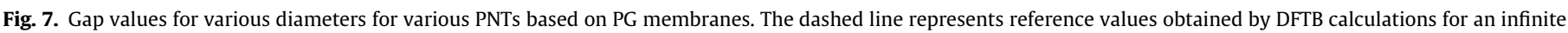

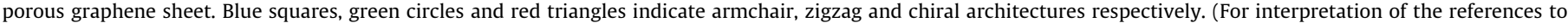
color in this figure legend, the reader is referred to the web version of this article.)

predicted by Eq. (4), obtained from ideal geometries and fixed bond sizes, and the values obtained from the optimized structures. For the PNTs studied, the PG type are slightly smaller by an average percent difference of $\sim 0.86$, while the graphenylene PNTs are slightly larger than the predicted value by a percent difference of $\sim 0.55$. Table 3 provides a comparison between the energies of 


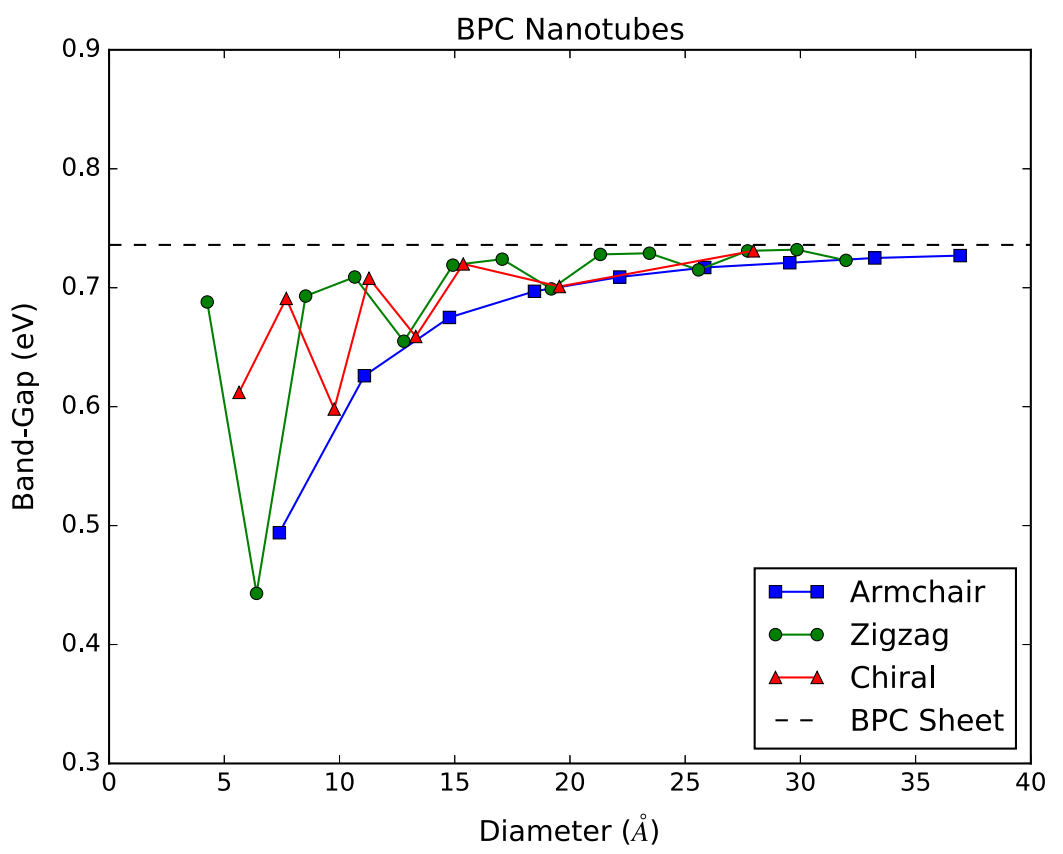

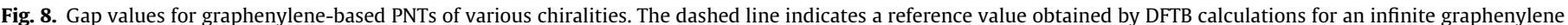

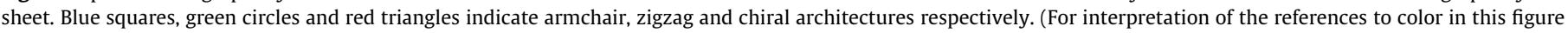
legend, the reader is referred to the web version of this article.)

usual carbon nanotubes and the proposed PNTs of the present work. In the case of PG-based PNTs, the original hexagonal pattern is preserved with some small distortions on bond distances that became longer for inter-hexagonal bonds (typically single bonds with $\sim 1.48 \AA$ ) if compared with the intra hexagonal bond sizes ( $\sim 1.4 \AA$ ). Representative PG-based PNTs are shown in Fig. 3. For graphenylene PNTs, bonds inside hexagons vary around two different values: $\sim 1.46 \AA$ and $\sim 1.38 \AA$, while butadiene bonds are typically close to $\sim 1.49 \AA$, the last value indicating single bonds. In both cases, PG based and graphenylene based PNTs, the variation on bond distances can be interpreted as the effect of different curvatures on the porous nanotubes.

MD simulations were used to determine if the PNTs would be stable above room temperature. Starting from the geometry determined in the optimization process, MD simulations at three different temperatures, namely $300 \mathrm{~K}, 600 \mathrm{~K}$ and $900 \mathrm{~K}$, were performed independently for at least $10 \mathrm{ps}$ for each one of the PNTs. Two representative cases of simulations carried at the higher temperature ( $900 \mathrm{~K}$ ) can be seen in the videos provided in the supplementary materials. These videos show nanotubes with lengths equivalent to several primitive cells of a graphenylene-based and a porous graphene-based PNT during a simulation that includes a small heating that goes from $300 \mathrm{~K}$ to $900 \mathrm{~K}$ and stabilizes at this final constant temperature of $900 \mathrm{~K}$. During the MD simulations, the studied PNTs presented high structural stability with no bond breaking, despite the presence of significant deformations caused by thermal agitation. This indicates that these materials would be structurally and chemically stable even at temperatures that are much higher than those typically supported by regular electronic devices.

\subsection{Electronic structure}

Charge distributions, shown in Fig. 4, reveal important differences between PG-based and graphenylene-based PNTs. In order to show representative cases, Fig. 4(a) and (c) present charge distributions for PG-based and BPC-based PNTs with chiral indices $(4,4)$.
The surfaces were plotted using an isosurface value of 0.01 with the VMD software [50]. The charge is nearly uniformly distributed over the $\mathrm{C}$ atoms in the graphenylene-based PNTs, whereas on the PG-based PNTs the charge is slightly more concentrated on the $C$ atoms then on the $\mathrm{H}$ atoms. The plots in Fig. 4(b) and (d) present a comparison between the total charge density and the density that would be obtained by summing up the densities of the neutral atoms in each region of the structures. These plots characterize charge transfers between atoms in the structure. Regions in which the total electronic density is smaller than that expected for the superposition of neutral atoms are colored in red, the opposite situation leads to a blue ${ }^{2}$ colored region. Fig. 4(b) shows that for the PG tubes, charge transfers from $\mathrm{H}$ to $\mathrm{C}$ atoms, while Fig. 4(d) indicates that the charge transfer is from $\mathrm{C}$ atoms to the region between them, due to the formation of $\mathrm{C}-\mathrm{C}$ bonds. For these plots, we considered an isosurface value of 0.05 .

Tables 1 and 2 show that the PNTs have band gaps, $\Delta \mathrm{E}$, similar to their two dimensional counterparts, preserving the semiconducting and insulating characteristics of graphenylene and PG sheets respectively. For instance, values given in Table 1 indicate a $\Delta \mathrm{E}$ in a range from $3.0 \mathrm{eV}$ to $3.5 \mathrm{eV}$ for $\mathrm{PG}$-based structures while the corresponding planar sheet presented a gap of $\sim 3.27 \mathrm{eV}$. On the other hand, graphenylene PNTs present gaps in the range $0.4 \mathrm{eV}$ to $0.75 \mathrm{eV}$ (Table 2), while the calculated gap for their planar counterpart (graphenylene sheet) was $\sim 0.74 \mathrm{eV}$. These are also different from the results reported elsewhere indicating metallic behavior in the case of nanotubes constructed with other kind of biphenylene-based planar structures, namely those with octagonal pores [27]. Representative band structure and density of states (DOS) plots are shown in Figs. 5 and 6, each one for three different chiralities. Armchair and chiral PG-based PNTs present indirect band gaps, while zigzag PNTs show direct gaps. Whereas for graphenylene-based PNTs, zigzag and armchair PNTs present direct band gaps while chiral ones can have either a direct or an

\footnotetext{
${ }^{2}$ For interpretation of color in Fig. 4, the reader is referred to the web version of this article.
} 

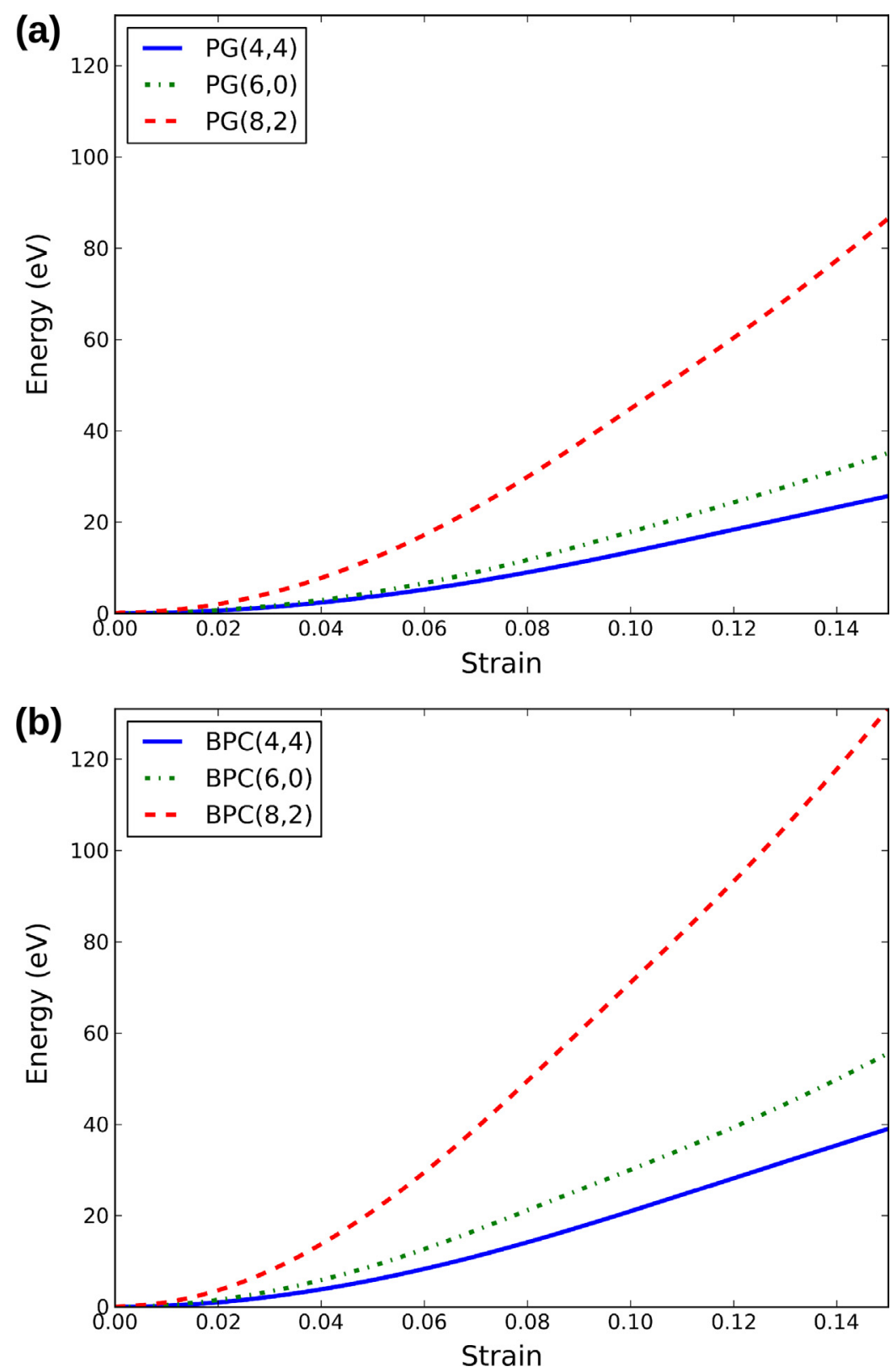

Fig. 9. Effect of axial strain in energy (relative to the zero-strain configuration) (a) PG-based PNTs. (b) Graphenylene-based PNTs.

indirect gap. These characteristics may be relevant for photoluminescence-related behavior of these structures [51].

For all PG-based PNTs we observe flat bands formed mainly by the combination of carbon's $2 p$ orbitals. Since the effective mass, $\mathrm{m}^{*}$, of a charge carrier (close to the top of valence band or bottom of conduction band) can be calculated by [52]

$m^{*}=\hbar^{2}\left(\frac{\partial^{2} E}{\partial k^{2}}\right)^{-1}$,

one can see that these materials present large effective masses, leading to low mobility. For this reason it is expected that PGbased PNTs will likely be insulators.

In Figs. 7 and 8 the dependencies between gap values and diameters are shown. The trends of each chirality type of each kind of PNT are similar. As the diameter of the PG-based PNTs increases the band gap decreases towards the value found for a planar PG sheet. Such trend is only violated by the 5Ådiameter PG-type
PNT. This may be interpreted as an effect due to the interaction between carbon's $2 p$ orbitals in the high strained structure. Conversely, graphenylene-based PNTs gaps increase in an oscillating fashion with growing tube diameters trending towards the value of a planar graphenylene sheet.

The bands formed by carbon $2 \mathrm{p}$ states in chiral graphenylenebased PNTs are flatter than the $2 \mathrm{p}$ states in armchair and zigzag graphenylene-based PNTs; see the $(6,2)$ graphenylene-based PNT in Fig. 6(e). This is due to the higher strained configuration of these tubes if compared to zigzag and armchair ones. These flat bands result in large effective masses for the charge carriers in both, the valence band (positive carriers) and in conduction band (negative carriers) indicating low carrier mobility despite the semiconducting band gap.

Another interesting effect to study is the influence of axial stress on the electronic structure of PNTs. We considered the effect of axial strain up to $\varepsilon=\Delta L / L \sim 0.15$ ( $\mathrm{L}$ is the tube length) on gap values for the PNTs considered in the present work. In all cases, 

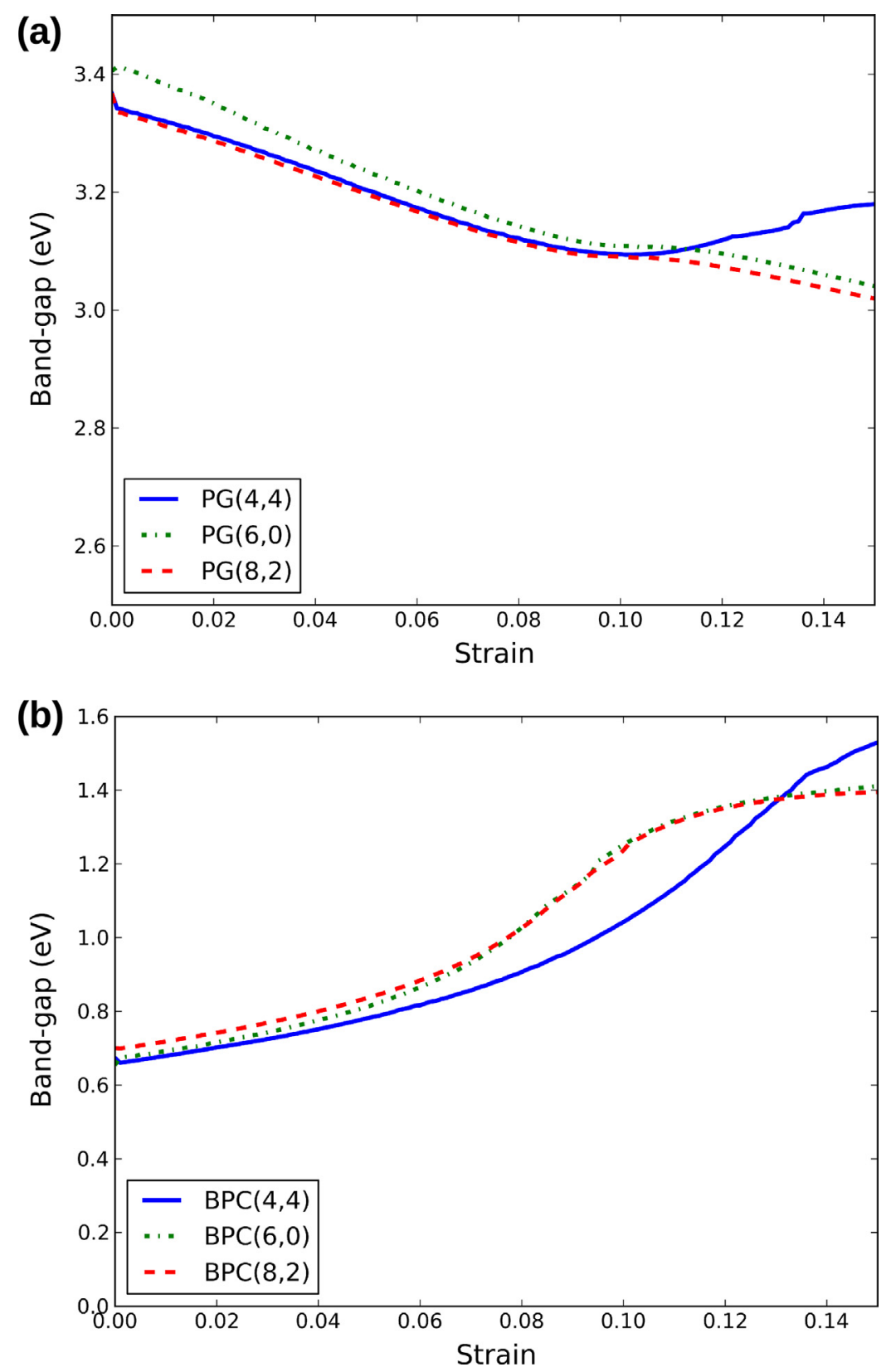

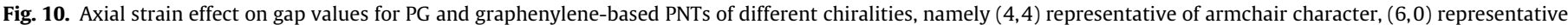
of zigzag and $(8,2)$ of chiral character. (a) PG-based PNTs. (b) Graphenylene-based PNTs.

the energy increases smoothly with axial strain as shown in Fig. 9. Fig. 10(a) shows our results for PG based nanotubes where one can see that, starting with $\varepsilon=0$, higher strain values correspond to smaller gap values with a monotonic decrease for $(6,0)$ and $(8,2)$ tubes. On the other hand, the $(4,4)$ tube presents a minimum at $\varepsilon \sim 0.1$ and the gap opening increases again for higher deformations, although the values still smaller than $3.2 \mathrm{eV}$ up to the maximum strain considered. Fig. 10(b) shows the influence of strain in the axial direction for graphenylene-based nanotubes, which present a reversed trend if compared to that found for PG based PNTs. Gap values for graphenylene-based PNTs increase monotonically with the increasing of strain. In both cases, higher strain (e.g. $\Delta L / L \sim 0.2$ ) causes plastic deformations of the PNTs, which coincide with abrupt changes in gap values (not shown).

Earlier studies considered usual CNTs [53,54] and reported a linear dependence between the gap opening and the applied axial strain. In the present work, we found that DFTB predicts a deviation from linearity in both cases. These deviations from linearity could be due to the fact that PNTs present several bonding types (double bonds, single bonds, C-H bonds) that weakens the structure and facilitate greater atomic rearrangement. In this context, the effect of band gap variation observed while strain is applied can be understood as a consequence (at least partially) of electronic interactions of atomic orbitals that result in some degree of repulsion combined with orbital recombinations that modify the band structure. In Fig. 11 it is possible to compare the projected density of states (PDOS) for representative porous nanotubes investigated in the present work. In that figure, it is possible to identify that $2 p$ states are the main contribution for the majority of states located close to the Fermi level (located at $0 \mathrm{eV}$ in the figure). This characteristics indicates that, upon deformations, the interaction and repulsion effects 

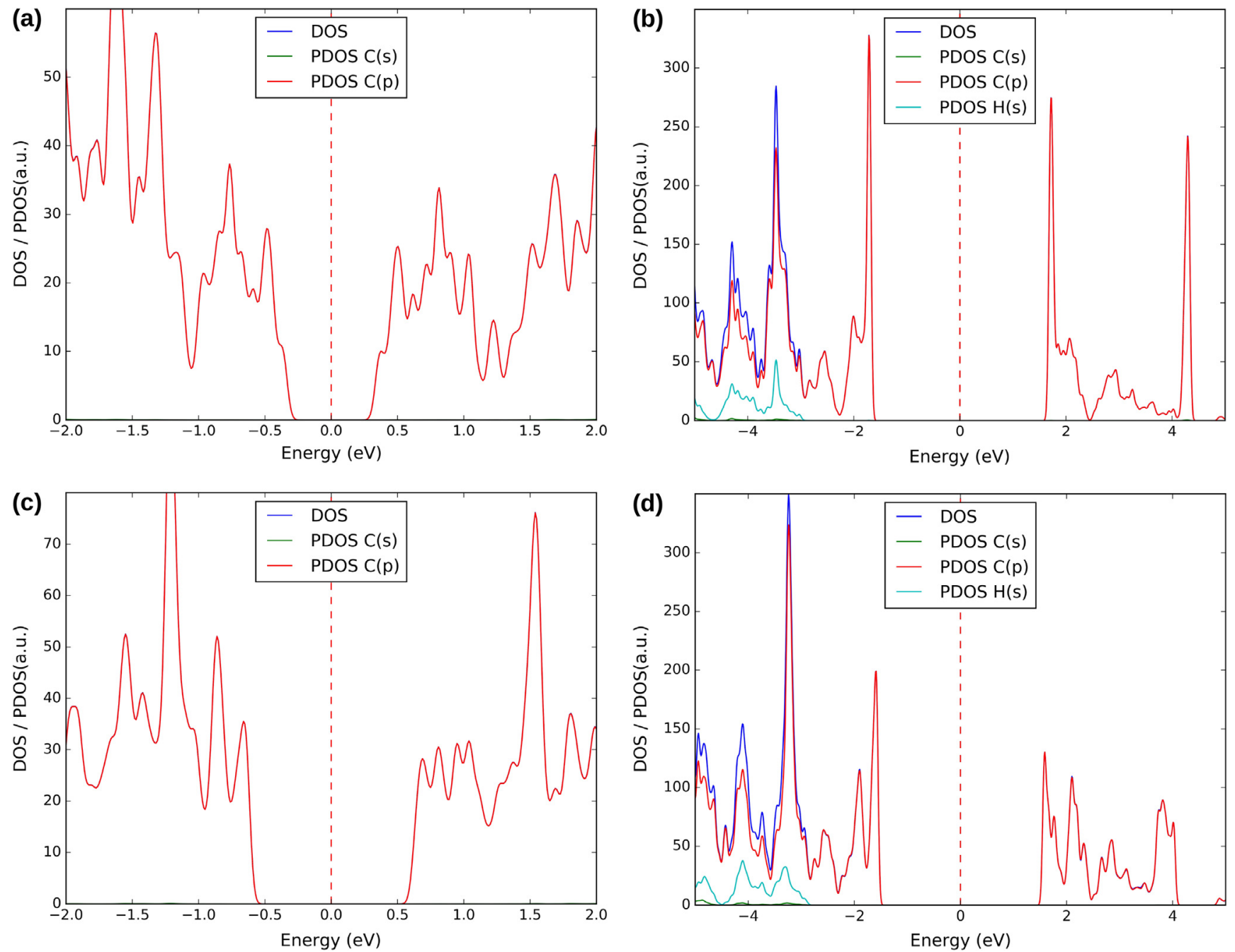

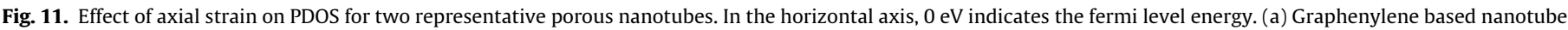

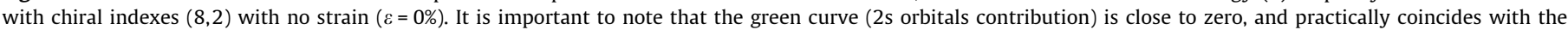

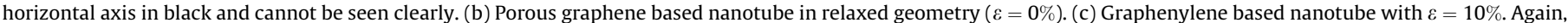

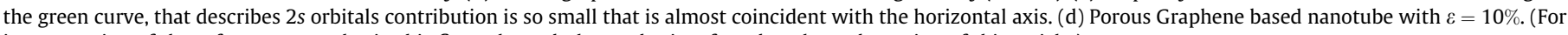
interpretation of the references to color in this figure legend, the reader is referred to the web version of this article.)

between $2 p$ states are the responsible for the gap modifications as described by Fig. 10 .

In order to evaluate the quality of results obtained in the DFTB approximation level, we carried some DFT calculations for representative porous nanotubes considering both types, PG and graphenylene based PNTs using different exchange-correlation potentials (PBE [55] and B3LYP [56,57]). Our calculations indicate that DFTB results are in relative accordance with DFT results in the sense that it predicts approximately the relative variation on gap values in both cases (PG-based and graphenylene-based). Also, DFTB predicts correctly the trends in these band gap openings, that is: an increasing gap with strain in the case of graphenylene-based tubes and decreasing gaps with strain for PG-based nanotubes. In terms of absolute values, DFTB predictions are closer to B3LYP results for the pure carbon porous nanotubes than PBE. This can be interpreted as a favorable result for DFTB if one considers calculations reported elsewhere by Goddard and coworkers [58] where they conclude that B3LYP provides the best description for the gap opening of carbon nanotubes. On the other hand, for porous graphene-based nanotubes, relative variations of bandgap openings predicted by DFTB approximation still in accordance with B3LYP and PBE, but absolute values are closer to PBE results.

\section{Conclusions}

We investigate the geometrical configuration and electronic structure of two new classes of nanotube architectures. The nanotubes were built using two different repeating units, the first based on porous graphene and the second based on graphenylene structure. Band gaps vary depending on tube diameters. Porous based tubes present higher band gaps than that found for PG nanosheets, while graphenylene-based tubes present gap values smaller than their two dimensional counterpart. Variation on the diameter of these tubes revealed a variation tendency on gap values of both nanotube types towards their respective planar sheets band gaps. Porous based nanotubes present a decrease in gap values and graphenylene type present increase of band gap values for increasing diameters. The influence of axial strain on the band gap opening was also investigated. Our results indicate that PG-based PNTs can have their band gap decreased by $\sim 10 \%$ in some cases. For graphenylene-based PNTs we found that strain application can induce a substantial increase on the band gap opening, that can reach up to $100 \%$ in some cases. Having these adjustable physical properties make these systems good candidates for the construction of nano-sensors or nano-devices. 


\section{Acknowledgments}

Nanotube models where produced using Greenwood: A library for creating molecular models and processing molecular dynamics simulations [59].

\section{Funding}

This work was supported in part by the Brazilian Agencies CNPq, CAPES, FAPESP. Ricardo Paupitz thanks the financial support of Fapesp (Grant 2014/15521-9) and CNPq (Grant 308298/2014-4).

\section{Appendix A. Supplementary material}

Supplementary data associated with this article can be found, in the online version, at http://dx.doi.org/10.1016/j.commatsci.2017. 09.009.

\section{References}

[1] K.S. Novoselov, A.K. Geim, S.V. Morozov, D. Jiang, Y. Zhang, S.V. Dubonos, I.V. Grigorieva, A.A. Firsov, Electric field effect in atomically thin carbon films, Science 306 (5696) (2004) 666-669.

[2] A.H. CastroNeto, F. Guinea, N.M.R. Peres, K.S. Novoselov, A.K. Geim, The electronic properties of graphene, Rev. Mod. Phys. 81 (1) (2009) 109-162.

[3] Z. Wang, C.P. Puls, N.E. Staley, Y. Zhang, A. Todd, J. Xu, C.A. Howsare, M.J. Hollander, J.A. Robinson, Y. Liu, Technology ready use of single layer graphene as a transparent electrode for hybrid photovoltaic devices, Phys. E: Lowdimensional Syst. Nanostruct. 44 (2) (2011) 521-524, http://dx.doi.org/ 10.1016/j.physe.2011.10.003. < <ttp://www.sciencedirect.com/science/ article/pii/S1386947711003651>.

[4] K.E. Whitener, Reversible graphene functionalization for electronic applications: a review, in: American Chemical Society, 2014, pp. 41-54, http://dx.doi.org/10.1021/bk-2014-1183.ch003 (Chapter4) <http://pubs.acs. org/doi/pdf/10.1021/bk-2014-1183.ch003> <http://pubs.acs.org/doi/abs/10. 1021/bk-2014-1183.ch003>.

[5] J.L. Achtyl, R.R. Unocic, L. Xu, Y. Cai, M. Raju, W. Zhang, R.L. Sacci, I.V. Vlassiouk, P.F. Fulvio, P. Ganesh, et al., Aqueous proton transfer across single-layer graphene, Nat. Commun. 6 (2015).

[6] J.T. Robinson, M.K. Zalalutdinov, C.E. Junkermeier, J.C. Culbertson, T.L. Reinecke, R. Stine, P.E. Sheehan, B.H. Houston, E.S. Snow, Structural transformations in chemically modified graphene, Solid State Commun. 152 (21) (2012) 19901998, http://dx.doi.org/10.1016/j.ssc.2012.04.051. www.sciencedirect.com/science/article/pii/S0038109812002578>.

[7] M.K. Zalalutdinov, J.T. Robinson, C.E. Junkermeier, J.C. Culbertson, T.L. Reinecke, R. Stine, P.E. Sheehan, B.H. Houston, E.S. Snow, Engineering graphene mechanical systems, Nano Lett. 12 (8) (2012) 4212-4218, http://dx.doi.org/ 10.1021/nl3018059, <http://pubs.acs.org/doi/pdf/10.1021/nl3018059> <http://pubs.acs.org/doi/abs/10.1021/nl3018059>.

[8] J.T. Robinson, M.K. Zalalutdinov, C.D. Cress, J.C. Culbertson, A.L. Friedman, A. Merrill, B.J. Landi, Graphene strained by defects, ACS Nano $0(0)(0)$ null, pMID: 28463478. http://dx.doi.org/10.1021/acsnano.7b00923.

[9] S.C. Hernndez, C.J.C. Bennett, C.E. Junkermeier, S.D. Tsoi, F.J. Bezares, R. Stine, J. T. Robinson, E.H. Lock, D.R. Boris, B.D. Pate, J.D. Caldwell, T.L. Reinecke, P.E. Sheehan, S.G. Walton, Chemical gradients on graphene to drive droplet motion, ACS Nano 7 (6) (2013) 4746-4755, http://dx.doi.org/10.1021/nn304267b, pMID: 23659463.

[10] V. Coluci, D. Galvao, A. Jorio, Geometric and electronic structure of carbon nanotube networks: 'super'-carbon nanotubes, Nanotechnology 17 (3) (2006) $617-621$.

[11] V.R. Coluci, R.P.B. dos Santos, D.S. Galvao, Topologically closed macromolecules made of single walled carbon nanotubes-'super'-fullerenes, J. Nanosci. Nanotechnol. 10 (7) (2010) 4378-4383.

[12] F. Ouyang, S. Peng, Z. Liu, Z. Liu, Bandgap opening in graphene antidot lattices: the missing half, ACS Nano 5 (5) (2011) 4023-4030.

[13] R. Paupitz, C.E. Junkermeier, A.C.T. van Duin, P.S. Branicio, Fullerenes generated from porous structures, PCCP 16 (46) (2014) 25515-25522, http://dx.doi.org/ 10.1039/c4cp03529a, <Go to ISI>://WOS:000344989500039.

[14] E. Perim, R. Paupitz, P.A.S. Autreto, D.S. Galvao, Inorganic graphenylene: a porous two-dimensional material with tunable band gap, J. Phys. Chem. C 118 (41) (2014) 23670-23674, http://dx.doi.org/10.1021/jp502119y, perim, E. Paupitz, R. Autreto, P.A.S. Galvao, D., <Go to ISI>://WOS:000343333600027.

[15] S. Stankovich, D.A. Dikin, R.D. Piner, K.A. Kohlhaas, A. Kleinhammes, Y. Jia, Y. $\mathrm{Wu}$, S.T. Nguyen, R.S. Ruoff, Synthesis of graphene-based nanosheets via chemical reduction of exfoliated graphite oxide, Carbon 45 (7) (2007) 15581565, http://dx.doi.org/10.1016/j.carbon.2007.02.034.

$<$ http:// www.sciencedirect.com/science/article/pii/S0008622307000917>.

[16] S. Gilje, S. Han, M. Wang, K.L. Wang, R.B. Kaner, A chemical route to graphene for device applications, Nano Lett. 7 (11) (2007) 3394-3398.
[17] R. Ruoff, Graphene: calling all chemists, Nat. Nanotechnol. 3 (1) (2008) $10-11$.

[18] J.O. Sofo, A.S. Chaudhari, G.D. Barber, Graphane: a two-dimensional hydrocarbon, Phys. Rev. B 75 (2007) 153401, http://dx.doi.org/10.1103/ PhysRevB.75.153401. <http://link.aps.org/doi/10.1103/PhysRevB.75.153401>.

[19] S. Ryu, M.Y. Han, J. Maultzsch, T.F. Heinz, P. Kim, M.L. Steigerwald, L.E. Brus, Reversible basal plane hydrogenation of graphene, Nano Lett. 8 (12) (2008) 4597-4602.

[20] D. Elias, R. Nair, T. Mohiuddin, S. Morozov, P. Blake, M. Halsall, A. Ferrari, D. Boukhvalov, M. Katsnelson, A. Geim, K. Novoselov, Control of graphene's properties by reversible hydrogenation: evidence for graphane, Science 323 (5914) (2009) 610-613.

[21] J.T. Robinson, J.S. Burgess, C.E. Junkermeier, S.C. Badescu, T.L. Reinecke, F.K. Perkins, M.K. Zalalutdniov, J.W. Baldwin, J.C. Culbertson, P.E. Sheehan, E.S. Snow, Properties of fluorinated graphene films, Nano Lett. 10 (8) (2010) 3001 3005, http://dx.doi.org/10.1021/nl101437p, <http://pubs.acs.org/doi/pdf/10. 1021/nl101437p> <http://pubs.acs.org/doi/abs/10.1021/nl101437p>.

[22] R.Paupitz, P.A.S. Autreto, S.B. Legoas, S.G. Srinivasan, T.van Duin, D.S. Galvao, Graphene to fluorographene and fluorographane: a theoretical study, Nanotechnology 24(3). http://dx.doi.org/10.1088/0957-4484/24/3/035706.

[23] C.E. Junkermeier, S.C. Badescu, T.L. Reinecke, , Highly fluorinated graphene 2014.

[24] D. Solenov, C. Junkermeier, T.L. Reinecke, K.A. Velizhanin, Tunable adsorbateadsorbate interactions on graphene, Phys. Rev. Lett. 111 (2013) 115502, http:// dx.doi.org/10.1103/PhysRevLett.111.115502. <http://link.aps.org/doi/10 1103/PhysRevLett.111.115502>.

[25] C.E. Junkermeier, D. Solenov, T.L. Reinecke, Adsorption of $\mathrm{NH}_{2}$ on graphene in the presence of defects and adsorbates, J. Phys. Chem. C 117 (0) (2013) 27932798, http://dx.doi.org/10.1021/jp309419x, <http://pubs.acs.org/doi/pdf/10. 1021/jp309419x><http://pubs.acs.org/doi/abs/10.1021/jp309419x>.

[26] M. Bieri, M. Treier, J. Cai, K. Ait-Mansour, P. Ruffieux, O. Groning, P. Groning, M. Kastler, R. Rieger, X. Feng, K. Mullen, R. Fasel, Porous graphenes: twodimensional polymer synthesis with atomic precision, Chem. Commun. (45) (2009) 6919-6921.

[27] M.A. Hudspeth, B.W. Whitman, V. Barone, J.E. Peralta, Electronic properties of the biphenylene sheet and its one-dimensional derivatives, ACS Nano 4 (8) (2010) 4565-4570, http://dx.doi.org/10.1021/nn100758h, pMID: 20669980.

[28] G. Brunetto, P.A.S. Autreto, L.D. Machado, B.I. Santos, R.P.B. dos Santos, D.S. Galvao, Nonzero gap two-dimensional carbon allotrope from porous graphene, J. Phys. Chem. C 116 (23) (2012) 12810-12813.

[29] A.L. Enyashin, A.N. Ivanovskii, Graphene allotropes, Phys. Status Solid B-Basic Solid State Phys. 248 (8) (2011) 1879-1883.

[30] E.H. Baughman, R.M. Kertesz, Structure-property predictions for new planar forms of carbon - layered phases containing sp2 and sp atoms, J. Chem. Phys. 87 (1987) 6687-6699.

[31] Q.-S. Du, P.-D. Tang, H.-L. Huang, F.-L. Du, K. Huang, N.-Z. Xie, S.-Y. Long, Y.-M Li, J.-S. Qiu, R.-B. Huang, A new type of two-dimensional carbon crystal prepared from 1,3,5-trihydroxybenzene, Sci. Rep. 7 (2017) 40796.

[32] R. Totani, C. Grazioli, T. Zhang, I. Bidermane, J. Lüder, M. DeSimone, M. Coreno B. Brena, L. Lozzi, C. Puglia, Electronic structure investigation of biphenylene films, J. Chem. Phys. 146 (5) (2017) 054705.

[33] F. Schlütter, T. Nishiuchi, V. Enkelmann, K. Müllen, Octafunctionalized biphenylenes: molecular precursors for isomeric graphene nanostructures, Angew. Chem. Int. Ed. 53 (6) (2014) 1538-1542.

[34] P.A. Denis, Stability and electronic properties of biphenylene based functionalized nanoribbons and sheets, J. Phys. Chem. C 118 (43) (2014) 24976-24982, http://dx.doi.org/10.1021/jp5069895.

[35] P.A. Denis, F. Iribarne, Hydrogen storage in doped biphenylene based sheets, Comput. Theor. Chem. 1062 (2015) 30-35, http://dx.doi.org/10.1016/ j.comptc.2015.03.012. <http://www.sciencedirect.com/science/article/pii/ S2210271X15001188>

[36] N. Chopra, R. Luyken, K. Cherre, V. Crespi, M. Cohen, S. Louie, A. Zettl, Boronnitride nanotubes, Science 269 (5226) (1995) 966-967, http://dx.doi.org/ $10.1126 /$ science.269.5226.966.

[37] S. Iijima, Helical microtubules of graphitic carbon, Nature 354 (6348) (1991) $56-58$.

[38] A.T. Koch, A.H. Khoshaman, H.D.E. Fan, G.A. Sawatzky, A. Nojeh, Graphenylene nanotubes, J. Phys. Chem. Lett. 6 (19) (2015) 3982-3987, http://dx.doi.org/ 10.1021/acs.jpclett.5b01707, pMID: 26722903.

[39] P. Koskinen, V. Mäkinen, Density-functional tight-binding for beginners, Comput. Mater. Sci. 47 (1) (2009) 237-253.

[40] M. Elstner, D. Porezag, G. Jungnickel, J. Elsner, M. Haugk, T. Frauenheim, S Suhai, G. Seifert, Self-consistent-charge density-functional tight-binding method for simulations of complex materials properties, Phys. Rev. B 58 (11) (1998) 7260-7268, http://dx.doi.org/10.1103/PhysRevB.58.7260. http://link. aps.org/doi/10.1103/PhysRevB.58.7260.

[41] D. Porezag, T. Frauenheim, T. Köhler, G. Seifert, R. Kaschner, Construction of tight-binding-like potentials on the basis of density-functional theory: application to carbon, Phys. Rev. B 51 (19) (1995) 12947-12957, http://dx. doi.org/10.1103/PhysRevB.51.12947.

[42] B. Aradi, B. Hourahine, T. Frauenheim, DFTB+, a sparse matrix-based implementation of the DFTB method, J. Phys. Chem. A 111 (26) (2007) 5678-5684.

[43] H. Manzano, A.N. Enyashin, J.S. Dolado, A. Ayuela, J. Frenzel, G. Seifert, Do cement nanotubes exist?, Adv Mater. 24 (24) (2012) 3239-3245, http://dx.doi. org/10.1002/adma.201103704. 
[44] T. Kubar, Z. Bodrog, M. Gaus, C. Kohler, B. Aradi, T. Frauenheim, M. Elstner, Parametrization of the SCC-DFTB method for halogens, J. Chem. Theory Comput. 9 (7) (2013) 2939-2949, http://dx.doi.org/10.1021/ct4001922.

[45] E. Rauls, J. Elsner, R. Gutierrez, T. Frauenheim, Stoichiometric and nonstoichiometric (1010) and (1120) surfaces in 2HSiC: a theoretical study, Solid State Commun. 111 (8) (1999) 459-464, http://dx.doi.org/10.1016/S00381098(99)00137-4. <http://www.sciencedirect.com/science/article/pii/ S0038109899001374>.

[46] C. Kohler, T. Frauenheim, Molecular dynamics simulations of $\{\mathrm{CFx}\}(\mathrm{x}=2,3)$ molecules at $\mathrm{Si}_{3} \mathrm{~N}_{4}$ and $\mathrm{SiO}_{2}$ surfaces, Surf. Sci. 600 (2) (2006) 453-460, http:// dx.doi.org/10.1016/j.susc.2005.10.044. www.sciencedirect.com/science/article/pii/S0039602805012379>.

[47] H.J. Monkhorst, J.D. Pack, Special points for brillouin-zone integrations, Phys Rev. B 13 (1976) 5188-5192, http://dx.doi.org/10.1103/PhysRevB.13.5188. $<$ http://link.aps.org/doi/10.1103/PhysRevB.13.5188>.

[48] J.-C. Charlier, X. Blase, S. Roche, Electronic and transport properties of nanotubes, Rev. Modern Phys. 79 (2) (2007) 677-732, http://dx.doi.org/ 10.1103/RevModPhys.79.677.

[49] M. Dresselhaus, G. Dresselhaus, R. Saito, Physics of carbon nanotubes, Carbon 33 (7) (1995) 883-891, http://dx.doi.org/10.1016/0008-6223(95)00017-8.

[50] W. Humphrey, A. Dalke, K. Schulten, VMD - visual molecular dynamics, J. Mol. Graph. 14 (1996) 33-38.

[51] H.R. Gutirrez, N. Perea-Lpez, A.L. Elas, A. Berkdemir, B. Wang, R. Lv, F. Lpez Uras, V.H. Crespi, H. Terrones, M. Terrones, Extraordinary room-temperature photoluminescence in triangular WS2 monolayers, Nano Lett. 13 (8) (2013) 3447-3454, http://dx.doi.org/10.1021/nl3026357, pMID: 23194096.
[52] S.H. Simon, The Oxford Solid State Basics, first ed., Oxford University Press, Great Clarendon Street, Oxford, Ox2 6DP, 2013.

[53] K.-M. Lin, Y.-H. Huang, W. Su, T. Leung, Strain effects on the band gap and work function of zigzag single-walled carbon nanotubes and graphene: a comparative study, Comput. Phys. Commun. 185 (5) (2014) 1422-1428, http://dx.doi.org/10.1016/j.cpc.2014.02.009. www.sciencedirect.com/science/article/pii/S0010465514000411>.

[54] J.W. Ding, X.H. Yan, J.X. Cao, D.L. Wang, Y. Tang, Q.B. Yang, Curvature and strain effects on electronic properties of single-wall carbon nanotubes, J. Phys.: Condens. Matter 15 (27) (2003) L439. <http://stacks.iop.org/0953-8984/15/i= $27 / \mathrm{a}=101>$.

[55] J.P. Perdew, K. Burke, M. Ernzerhof, Generalized gradient approximation made simple, Phys. Rev. Lett. 77 (1996) 3865-3868, http://dx.doi.org/10.1103/ PhysRevLett.77.3865. <https://link.aps.org/doi/10.1103/PhysRevLett.77. 3865>.

[56] A.D. Becke, Density functional thermochemistry. III. The role of exact exchange, J. Chem. Phys. 98 (7) (1993) 5648-5652, http://dx.doi.org/ $10.1063 / 1.464913$.

[57] P. Stephens, F. Devlin, C. Chabalowski, M.J. Frisch, Ab initio calculation of vibrational absorption and circular dichroism spectra using density functional force fields, J. Phys. Chem. 98 (45) (1994) 11623-11627.

[58] Y. Matsuda, J. Tahir-Kheli, W.A. Goddard III, Definitive band gaps for singlewall carbon nanotubes, J. Phys. Chem. Lett. 1 (19) (2010) 2946-2950.

[59] C.E. Junkermeier, Greenwood: A Library for Creating Molecular Models and Processing Molecular Dynamics Simulations. <https://github.com/ cjunkermeier/greenwood>. 